\title{
Nitric Oxide (NO) and Alpha-Tubulin Nitrotyrosination: A Possible Role in the Pathogenesis of Depression? Proposal for Concomitant Voltammetric and Molecular Biology Study
}

\author{
Francesco Crespi* \\ voltammetry Lab, Medical Research Centre, Italy \\ *Corresponding author: Francesco Crespi, voltammetry Lab., Medical Research Centre, Verona, Italy
}

\begin{tabular}{|c|c|}
\hline ARTICLE INFO & ABSTRACT \\
\hline Received: 蔧 August 07, 2020 & Nitric Oxide (NO) is a free radicalinvolved in diverse cellular functions, it also mediates \\
\hline Published: 幽 September 14, 2020 & $\begin{array}{l}\text { cellular damage through direct reaction with cell proteins or through generation of a } \\
\text { number of chemical products such as peroxynitrite ONOO-A number of works suggest }\end{array}$ \\
\hline & SSRI such as paroxetine act as NOS inhibitor. Our hypothesis is that the increase in \\
\hline ho) and Alpha-Tubulin Nitrotyrosina- & S observed after stressful conditions may be followed by intracellular NO incl \\
\hline on: A Possible Role in the Pathogenesis & nitrotyrosination which is irreversible and result in the block of the tyrosination/ \\
\hline & detyrosination cycle and to the microtubular dysfunction that may be responsible for \\
\hline tudy Biomed I Sci \& Tech Res 30(3)-202 & the observed neuronal plasticity failure (atrophy, cell death and neurogenesis decrease) \\
\hline 3JSTR. MS.ID.004940. & $\begin{array}{l}\text { in response to stress and depression. The present Research Proposal aims to verify } \\
\text { such hypothesis that could lead to potential new therapeutic indication for SSRI i.e. the }\end{array}$ \\
\hline $\begin{array}{l}\text { Abbreviations: NO: Nitric Oxide; TTC } \\
\text { Tubulin Tyrosine Carboxypeptidase; TT } \\
\text { Tubulin Tyrosine Ligase }\end{array}$ & $\begin{array}{l}\text { modulation of NOS activities and therefore modulation of NO levels within abnormal } \\
\text { conditions in which the increase of NO activity would influence negatively cell functions } \\
\text { in particular at the level of cytoskeleton. }\end{array}$ \\
\hline
\end{tabular}

\section{Mini Review}

\section{Nitric Oxide (NO)}

Nitric Oxide (NO) is a free radical produced from L-arginine in a reaction catalyzed by a set of related enzymes known as NO synthetases (NOS).NO is involved in diverse cellular functions, such as long-term potentiation, macrophage cytotoxicity, vasodilatation, and neurotransmission.This free radical also mediates cellular damage through direct reaction with cell proteins or through generation of several chemical products such as peroxynitrite (ONOO-), which mediates much of NO's cytotoxicity.One of the products of NO-derived reactions is nitro tyrosine, which is elevated in a number of pathological conditions $[1,2]$.

\section{Tubulin and Nitrotyrosination}

Microtubules are a candidate for nitro tyrosine-mediate protein dysfunction.It is well known that microtubules are spatially and functionally associated with a wide range of eukaryotic cell activities such as cell division, motility and transport, maintenance of cell shape and signal trasduction.Tubulin, the building block of microtubules, is a heterodimer composed of $\alpha$ - and $\beta$ - Tubulin. $\alpha$-Tubulin is subject to the post-traslational C-terminal removal and subsequent addition of tyrosine in reactions catalyzed by two enzymes: tubulin tyrosine carboxypeptidase (TTCP) and Tubulin tyrosine ligase (TTL). Recently it has been demonstrated that the modified amino acid Nitro tyrosine is post-translationally incorporated onto $\alpha$-Tubulin, in several cell types, leading to cell dysfunction and apoptosis [3,4].

\section{NO, Stress and Depression}

Up-regulation of the neuronal NOS (nNOS) expression has been shown in response to stressful conditions in various brain areas following stressful conditions such as restraint [5-7]. In addition, a 
number of works suggest a role of NO in psychiatric illness such as schizophrenia and anxiety disorders [8-10].[11] have reported for the first time that an SSRI (Paroxetine) is a NOS inhibitor in vivo and in vitro. Accordingly, we have observed that the SSRI fluoxetine could reduce NO levels when recorded in vivo in the striatum of anaesthetised rats [12].

\section{Tubulin and Depression}

We have previously demonstrated that in rat submitted to an animal depression model (Forced Swimming Test [FST]) the brain tyrosinated $\alpha$-Tubulin expression and 5-HT levels significantly decrease $(\mathrm{p}<0.01)$ about $20 \%$ and $40 \%$ respectively[13].We have also demonstrated using acute and sub-chronic restraint stress as well as various pharmacological treatments that parallel variation of the activity of these two systems is directly correlated [13-15]. Indeed, our results suggest that the 5-HT system may exert a role of modulator on the microtubular network dynamics that in the case of depression may lead to an observed consequent reduction of the neuronal plasticity events $[16,17]$. Additionally, the observation that NOS inhibitors such as SSRI (i.e. fluoxetine) influence either cerebral serotoninergic as well asNO levels [12]may lead to consider the presence of a complex interaction between these three systems and therefore to consider that inadequate production NO may also be implicated in stress and depression.

\section{Hypothesis}

In particular our hypothesis is that the increase in nNOS observed after stressful conditions [5-7] may be followed by intracellular NO increase. This increase could lead to a major production of ONOO- and consequently to the $\alpha$-tubulin nitro tyrosination. The nitro tyrosination of $\alpha$-tubulin is irreversible and result in the block of the tyrosination/detyrosination cycle $[3,18]$. This microtubular dysfunction may be responsible for the observed neuronal plasticity failure (atrophy, cell death and neurogenesis decrease) in response to stress and depression [16,17]. Among the various stress-related brain areas the hippocampus seems to be the most vulnerable to stressful conditions. In particular a chronic restraint stress cause atrophy of apical dendrites in CA3 pyramidal neurons of the hippocampus $[19,20]$. It is important to underline that the antibody specific for the tyrosinated $\alpha$-tubulin used in our works (clone TUB-1A2) is incapable to bind the nitrotyrosinated $\alpha$-tubulin [3]. These findings can suggest that the observed decrease of tyrosinated $\alpha$-tubulin expression subsequent to FST may be more related to the nitrotyrosination of the protein than to a reduced amount of tyrosinated $\alpha$-tubulin.In order to scrutiny our hypothesis 2 experiments could be done:

\section{Experiment 1: $\alpha$-Tubulin Tyrosination and Nitrotyrosination expression in rat brain areasafter acute and chronic restraint stress}

Rats (200-250g) will be submitted to acute (one day) or chronic (seven days) daily restraint periods of $2 \mathrm{~h}$ (group 1 ) or 6h (group 2) repetitively in a cylindrical chamber. Control rats will remain undisturbed in their cages.According to de Oliveira et al. [5], the animals will be sacrificed twenty-four hours after the last immobilization period. The brain will be rapidly removed, hippocampus, cortex and striatum dissected and then processed for a Western Blot method [13-15]allowing to analyze the expression of: I)total $\alpha$-tubulin II) tyrosinated $\alpha$-tubulin III) nitrotyrosination $\alpha$-tubulin.

\section{Experiment 2: In vivo No level in freely moving rat submitted to restraint stress andex vivo $\alpha$ - Tubulin Tyrosination and Nitrotyrosination expression}

Rats (200-250g) will be prepared as previously described for in vivo amperometric measurement of NO levels in specific brain areas, by means of specifically treated carbon fiber microelectrodes (CFE)[21,22].The electrochemical recordings will be taken before (control period), during and after the restraint stress. The restraint stress will be made as described above in a cylindrical chamber modified to permit the electrochemical recordings. Twenty-four hours after the last immobilization period the animals will be sacrificed the brain removed and processed for Western Blot Analysis.

\section{Experimental Procedure}

The objectives of this work will be challenged via various approaches. In particular:

a. Forced swimming test (FST) or other animal stress models $[14,15,23]$ to obtain the stress-induced cytoskeletal changes observed in our previous studies $[13,15]$.

b. In vivo voltammetry associated with carbon fibermicro biosensors $[24,25]$ to monitor NO levels in animals submitted to the behavioral models mentioned above.

c. Electrophysiological analysis of cell firing in various brain areas [26] this will allow the monitoring of 5-HT cell activity, the parallel detection via concomitant voltammetry measurements of serotonergic release [27]will indicate the putative correlation between the NO and the 5-HT systems in the experimental conditions applied. In particular the data gathered will add value to the hypothesis of an interrelationship between these two cerebral systems within the induced pathological conditions.

d. Molecular biology investigation to analyse the tyrosinated and nitrotyrosinated

e. $\alpha$-tubulin expression by means of the Western Blot method [15]

f. Pharmacological studies to analyse the effect of the SSRI upon NO production and nitrotyrosinated $\alpha$-tubulin expression $[24,28,29]$ 


\section{Conclusion}

Data gathered will be of help in supporting the hypothesis that the microtubular network is a potential new pathway that may increase our understanding of stress-related events. In addition, while confirming the involvement of the serotoninergic system in such pathologic conditions, they will propose a potential new therapeutic application for SSRIs i.e. the modulation of NOS activities and therefore that of NO levels within abnormal states in which the increase of NO activity would influence negatively cell functions in particular at the level of cytoskeleton.

\section{References}

1. Beckman JS, WH Koppenol (1996) Nitric oxide, superoxide, and peroxynitrite: the good, the bad, and ugly Cell Physiology 271(5): 14241437.

2. P Pacher, JS Beckman, L Liaudet (2007) Nitric Oxide and Peroxynitrite in Health and Disease. Physiological Reviews 87(1): 315-424.

3. JP Eiserich, AG Estevez, TV Bamberg, YZ Ye, PH Chumley, et al. (1999) Microtubule dysfunction by posttranslational nitrotyrosination of $\alpha$-tubulin: A nitric oxide-dependent mechanism of cellular injury. PNAS 96(11): 6365-6370.

4. G Ferrer Sueta, N Campolo, M Trujillo, S Bartesaghi, S Carballal, et al. (2018) Biochemistry of Peroxynitrite and Protein Tyrosine Nitration Chem Rev 118 (3): 1338-1408.

5. RMW de Oliveira, EA Del Bel, MLN Mamede Rosa, CM Padovan, JFW Deakin, et al. (2000) Expression of neuronal nitric oxide synthase mRNA in stress-related brain areas after restraint in rats. Neuroscience Letters 289(2): 123-126.

6. A Gądek Michalska, J Tadeusz, P Rachwalska, J Spyrka, J Bugajski (2012) Effect of repeated restraint on homotypic stress-induced nitric oxide synthases expression in brain structures regulating HPA axis. Pharmacological Report 64(6): 1381-1390.

7. C Vila Verde, ALZ Marinho, SF Lisboa, FS Guimarães (2016) Nitric oxide in the prelimbic medial prefrontal cortex is involved in the anxiogeniclike effect induced by acute restraint stress in rats. Neuroscience 320: 30-42.

8. BH Harvey (1996) Affective Disorders and Nitric Oxide: A Role in Pathways to Relapse and Refractoriness? Human Psychopharmacology 11(3): 309-319.

9. N Kordjazy, A Haj Mirzaian, S Amiri, S Ostadhadi, M Kordjazy, et al. (2015) Elevated level of nitric oxide mediates the anti-depressant effect of rubidium chloride in mice. European Journal of Pharmacology 762(5): 411-418.

10. SJ Brand, M Moller, BH Harvey (2015) A Review of Biomarkers in Mood and Psychotic Disorders: A Dissection of Clinical vs. Preclinical Correlates. Current Neuropharmacology 13(3): 324-368.

11. MS Finkel, F Laghrissi Thode, BG Pollock, J Rong (1996) Paroxetine is a novel nitric oxide synthase inhibitor. Psychopharmacology Bulletin 32(4): 653-658.

12. F Crespi (2010a) The selective serotonin reuptake inhibitor fluoxetine reduces striatal in vivo levels of voltammetric nitric oxide (NO): A feature of its antidepressant activity? Neuroscience Letters 470: 95-99.
13. F Crespi, M Bianchi (2002) Serotonin and neuronal plasticity relationship: a new mechanism involved in depression? European Neuropsychopharmacology 12: 191.

14. M Bianchi, C Heidbreder, F Crespi (2003) Cytoskeletal changes in the hippocampus following restrain stress: role of serotonin and microtubules. Synapse 49: 188-194.

15. F Crespi (2010b) Further Electrochemical and Behavioural Evidence of a Direct Relationship Between Central 5-HT and Cytoskeleton in the Control of Mood. The Open Neurology Journal 4: 5-14.

16. R Duman, J Malberg, S Nakagawa C D’S (2000) Neuronal plasticity and survival in mood disorders. Biological Psychiatry 48(8): 732-739.

17. EB Ansell, K Rando, K Tuit, J Guarnaccia, R Sinha (2012) Cumulative Adversity and Smaller Gray Matter Volume in Medial Prefrontal, Anterior Cingulate, and Insula Regions. Biological Psychiatry 72(1): 57-64.

18. AD Phung, K Soucek, L Kubala, RW Harper, JC Bulinski, et al. (2006) Posttranslational nitrotyrosination of $\alpha$-tubulin induces cell cycle arrest and inhibits proliferation of vascular smooth muscle cells. European Journal of Cell Biology 85(12): 1241-1252.

19. AM Magariños, JM García Verdugo, BS Mc Ewen (1997) Chronic stress alters synaptic terminal structure in hippocampus. PNAS 94(25): 14002-14008.

20. RJ Schloesser, DV Jimenez, NF Hardy, D Paredes, BJ Catlow, et al. (2014) Atrophy of pyramidal neurons and increased stress-induced glutamate levels in CA3 following chronic suppression of adult neurogenesis. Brain Struct. Funct 219: 1139-1148.

21. F Crespi (2002a) In vivo voltammetry and concomitant electrophysiology at a single biosensor to analyse ischaemia, depression and drug dependence. Journal of Neuroscience Methods 119: 173-184.

22. F Crespi (2014) Invasive or Non-Invasive Techniques and Sensors for Real Time In Vivo Sensing in the Brain" chapter in the book, Laboratory and Clinical Research: "Microelectrodes: Techniques, Structures for Biosensing and Potential Applications" Nova Science Publishers.

23. RD Porsolt, M Le Pichon, M Jalfre (1977) Depression: a new animal model sensitive to antidepressant treatments. Nature 266: 730-732.

24. F Crespi, M Campagnola, A Neudeck, K Mc Millan (2001) Can voltammetry measures nitrogen monoxide (NO) and/or nitrites? Journal of Neuroscience Methods 109 (59-70).

25. Z Rossetti, F Crespi (2004) Inhibition of Nitric Oxide release in vivo by ethanol Alcoholism: Clinical and Experimental Research 28: 1-6.

26. F Crespi (2002b) In vivo voltammetry and concomitant electrophysiology at a single biosensor to analyse ischaemia, depression and drug dependence. Journal of Neuroscience Methods 119: 173-184.

27. F Crespi (2013) Serotonin, how to find it...Review Article Xjenza Online. Journal of Malta Chamber of Scientists.

28. F Crespi (2010c) Is a divergent central serotonergic activity responsible for either despair or learning behavior in intact Wistar or Sprague-Dawley CD rats, respectively? A concomitant behavioral and electrochemical analysis. PSYCHOLOGY 1: 1-11.

29. F Crespi, Z Rossetti (2004) Pulse of Nitric Oxide Release in Response to Activation of N-Methyl-D-Aspartate Receptors in the Rat Striatum: Rapid Desensitisation, Inhibition by Receptor Antagonists and Potentiation by Glycine. J Pharmacol Exp Ther 309: 462-468. 
ISSN: 2574-1241

DOI: $10.26717 /$ BJSTR.2020.30.004940

Francesco Crespi. Biomed J Sci \& Tech Res

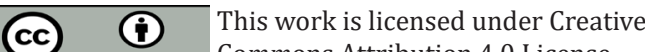

Submission Link: https://biomedres.us/submit-manuscript.php

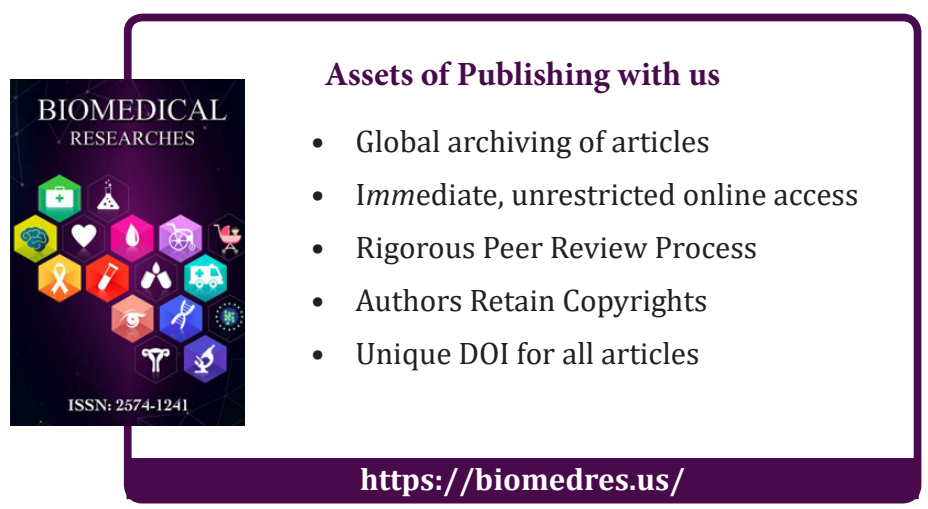

